Supporting Information for:

\title{
Pre-Seeded Optical Scatterers as a Template for Enhancing Order in Inorganic Phototropic Growth
}

\author{
Ethan Simonoff ${ }^{\dagger}, \ddagger$ Jonathan R. Thompson ${ }^{\S,}$, Madeline C. Meier ${ }^{\dagger}$, Kathleen Kennedy, \\ Kathryn R. Hamann ${ }^{\dagger}$, Nathan S. Lewis ${ }^{\dagger, l, *}$ \\ ${ }^{\dagger}$ Division of Chemistry and Chemical Engineering \\ $\S$ Division of Engineering and Applied Sciences \\ 'Beckman Institute \\ *These authors contributed equally \\ California Institute of Technology \\ Pasadena, CA 91125
}

*Corresponding Author: nslewis@,caltech.edu 


\section{Contents}

S1. Fourier analysis and peak-fitting methodology

S2. Cross-sectional SEM images of phototropically grown Se-Te films

S3. Simulated phototropically grown Se-Te on non-templated Pt substrates 


\section{S1. Fourier analysis and peak-fitting methodology}
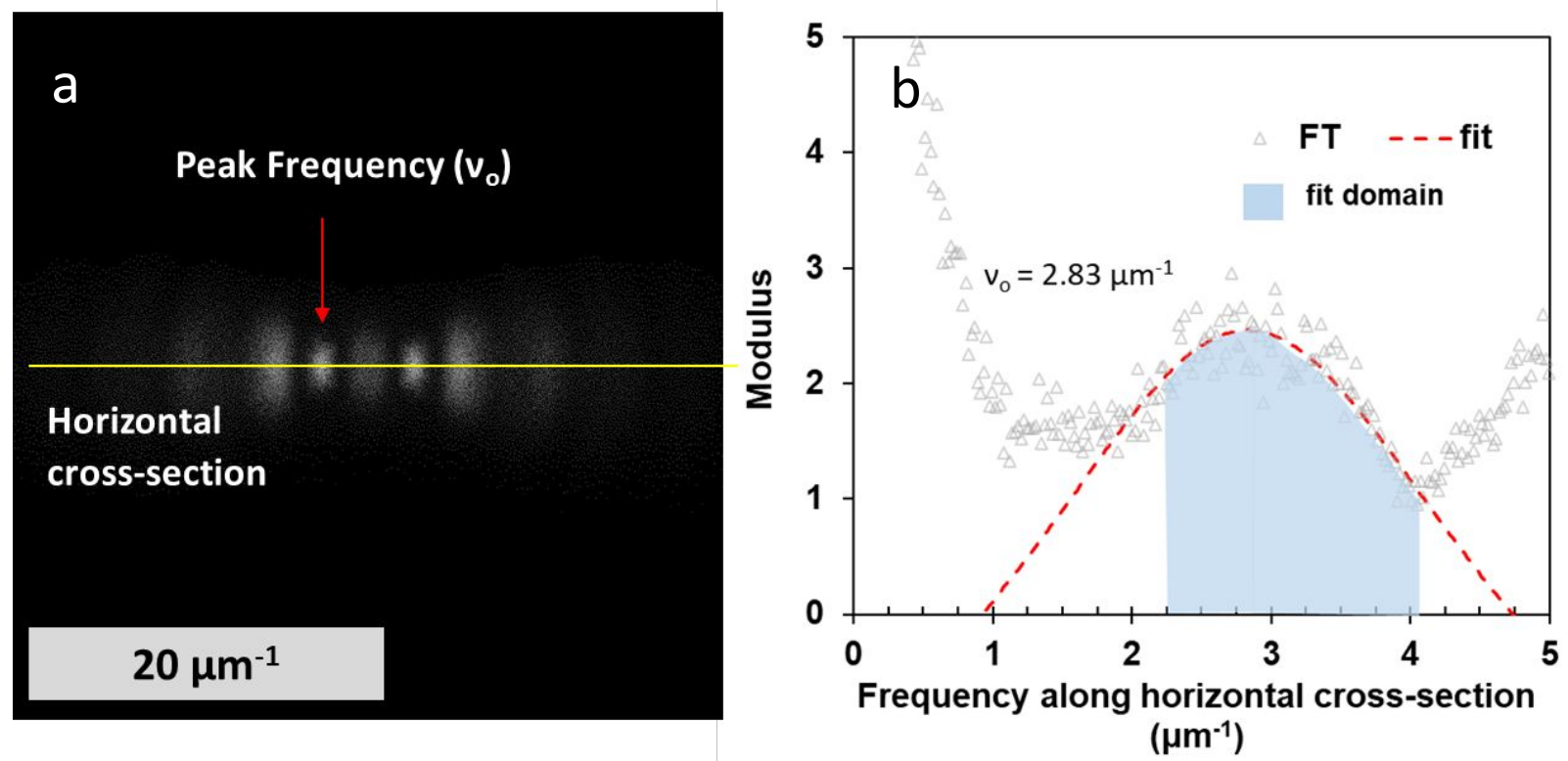

Figure S1: Pattern period was measured via analysis of the primary peaks in the 2D Fourier transform (FT) spectra of low-magnification SEM images. An example 2D FT spectrum is provided in (a). An associated plot of the horizontal (perpendicular to illumination polarization) cross-section through the origin, integrated vertically over 30 pixels, is provided in (b). Pattern period was defined as the average inter-lamellar spacing in phototropically grown films and was measured by determining and inverting the centroid frequency, $v_{o}$, of the primary peak. As demonstrated in (b), the peaks in the 2D FT were fitted using a Lorentzian function. The fit domain was chosen to minimize the residual between the peak intensity and the fitted curve. 

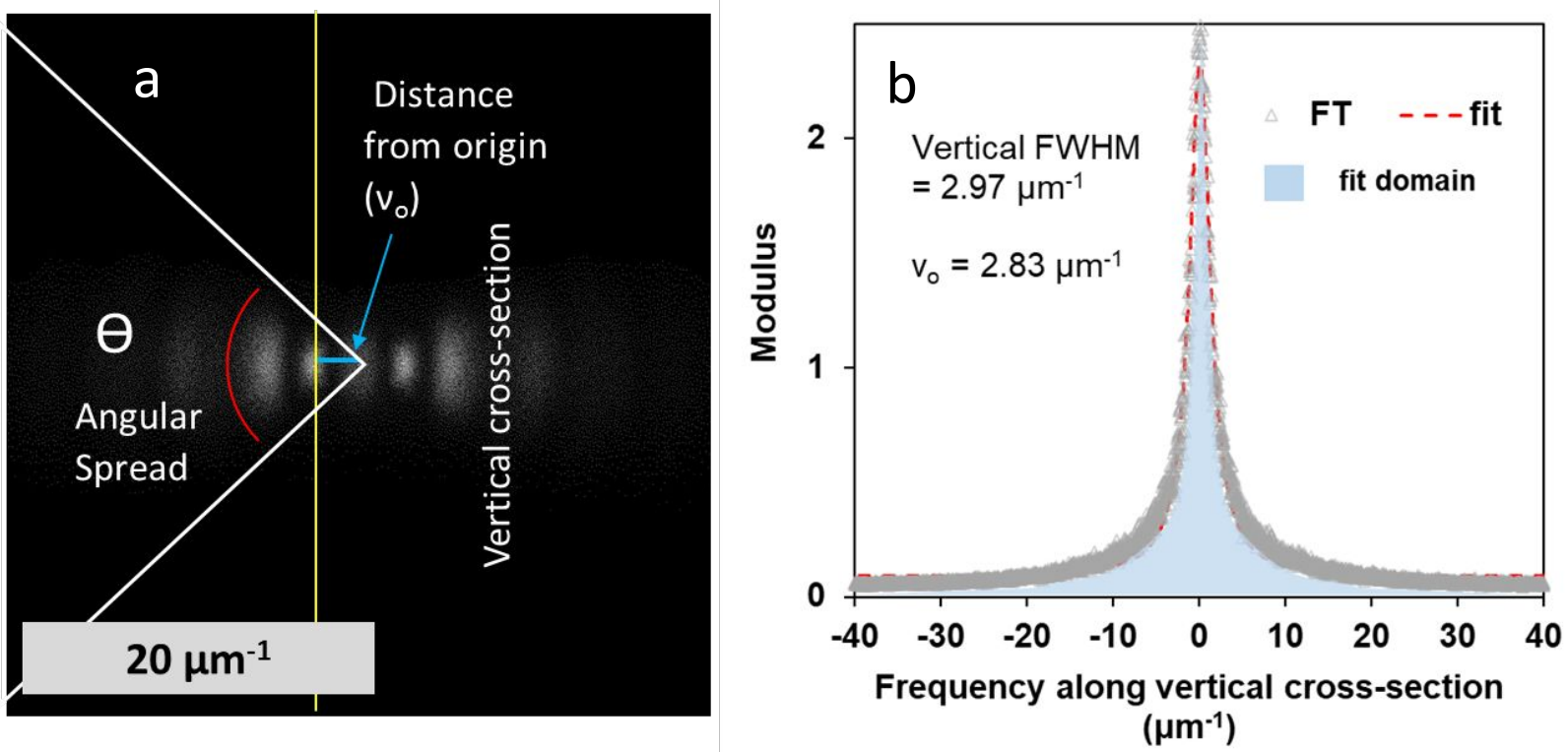

Figure S2: Pattern fidelity in phototropically grown films was quantified by using a figure-ofmerit calculated as the vertical (parallel to illumination polarization) full width at half maximum (FWHM) of the primary peak in the 2D FT spectra of low-magnification SEM images of respective films. An example 2D FT is provided in (a) and the associated surface profile is provided in (b). As in Figure S1, a Lorentzian function was used to fit 2D FT peak intensity. Using Equation S1, the frequency value of the vertical FWHM was converted to radial coordinates to yield the angular FWHM. Phototropically grown films demonstrating higher pattern fidelity consistently demonstrated narrower vertical FWHM.

Equation S1: $\quad$ Vertical FWHM $\left(^{\circ}\right)=\mathbf{2} \tan ^{-\mathbf{1}}\left(\frac{\text { Vertical FWHM }}{\mathbf{2} \cdot \boldsymbol{v}_{o}}\right)$ 
S2. Cross-sectional SEM images of phototropically grown Se-Te films

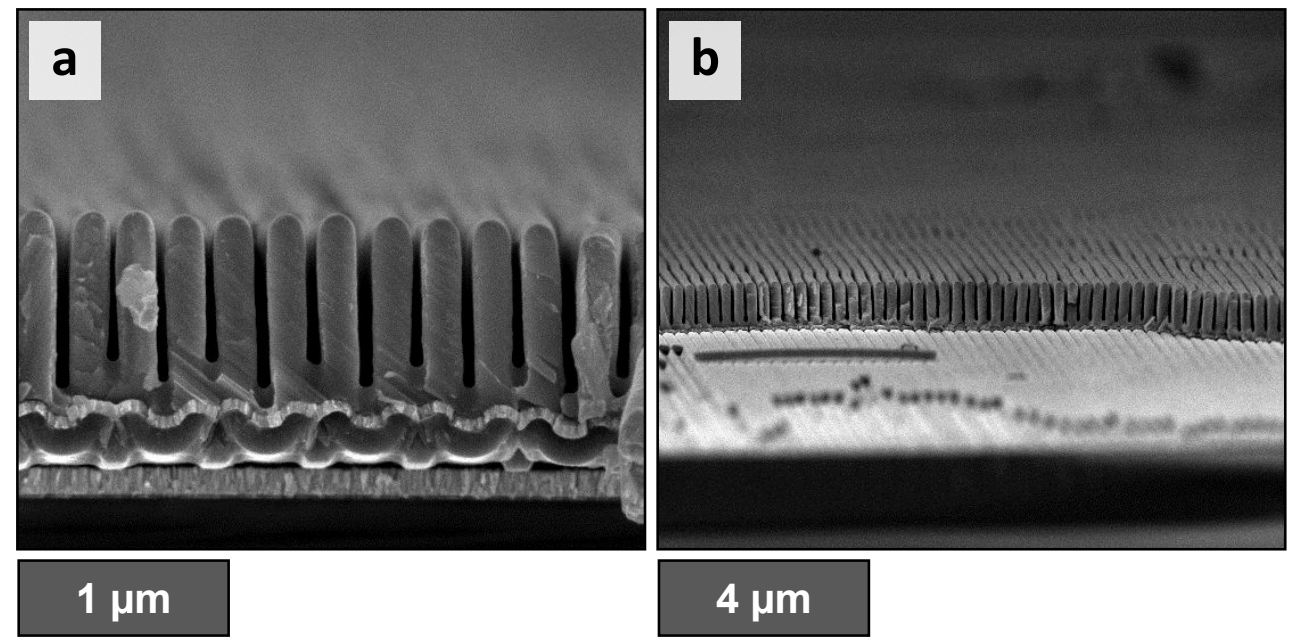

Figure S3: Representative cross-sectional SEM images of a phototropically grown Se-Te film deposited on physically templated Pt-coated $n^{+}-S i$ substrates with a Ti interlayer at (a) high and (b) low magnification. The film was grown under polarized $630 \mathrm{~nm}$ excitation from a narrow-band LED. 
S3. Simulated phototropically grown Se-Te on non-templated Pt substrates
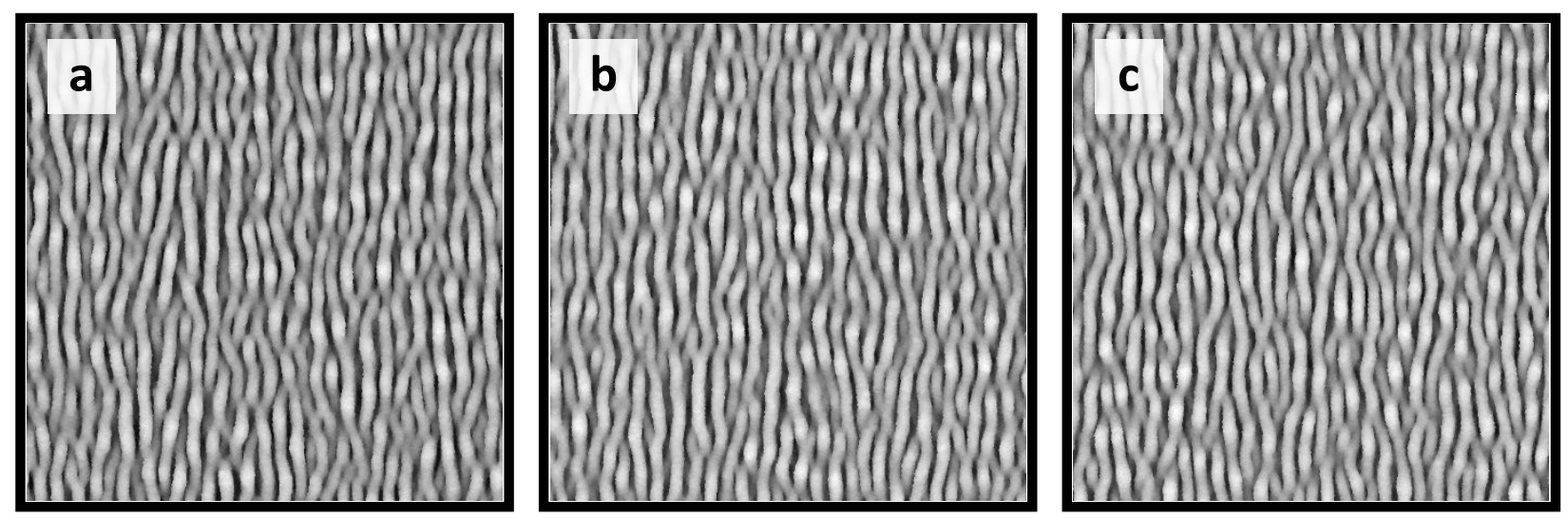

\section{$6 \mu \mathrm{m}$}

Figure S4: Simulated phototropic growth on non-templated Pt substrates with vertically polarized

illumination with $\lambda=630 \mathrm{~nm}$. Simulations were performed with periodic boundary conditions. (a), (b), and (c) are simulated under identical conditions and were used to demonstrate reproducibility and measure pattern period. Following the procedure in Figure S1, the average pattern period for these simulated patterns was $239 \pm 13 \mathrm{~nm}$, in excellent agreement with the expected trend of $\sim 242$ nm. 